\title{
Ionization efficiency and effusive delay time characterization of high temperature target-ion sources for RIB generation
}

\author{
R.F. Welton', G.D. Alton ${ }^{b}$, B. Cui and S.N. Murray ${ }^{b}$ \\ ${ }^{6}$ Oak Ridge National Laboratory, P.O. Box 2008, Oak Ridge, TN 3783-6368, USA \\ - Oak Ridge Institute of Science and Engineering, Oak Ridge, TN 3783 \\ 'China Institute fo Atomic Energy, Beijing, China
}

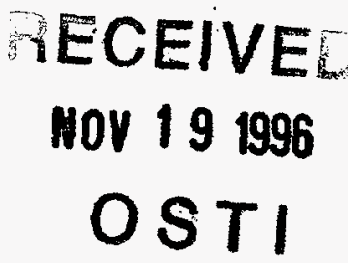

\begin{abstract}
Ion sources for radioactive ion beam (RIB) generation must efficiently ionize short-lived-radioactive nuclei released from on-line targets with minimal delay times. Delay times attributable to interactions between chemically active species and surfaces of the vapor transport system which are long compared to the half-life of the desired radioactive atom and/or low ionization efficiency of the targetion source (TIS) will result in a severe reduction of the RIB intensity available for research. We have developed complementary off-line techniques for directly measuring both effusive delay times and ionization efficiencies for chemically active species in high temperature TISs using only the stable complements of the radioactive element of interest. Equipment, designed and developed for these measurements, include: a high-temperature Ta valve; a differentially cooled injection nozzle; and a gaseous flow measurement and control system. These techniques are employed in a systematic investigation of fluorine transport and ionization in an electron-beam-plasma target/ion source (EBPTIS) designed for initial use at the Holifield Radioactive Ion Beam Facility (HRIBF).
\end{abstract}

\section{INTRODUCTION}

Off-line techniques have been developed for ionization efficiency and effusive delay time measurements for chemically reactive species in high temperature target/ion sources (TISs). Molecular feed materials, containing a large fraction of the reactive atom of interest, are introduced into the TIS at well defined flow rates and time distributions. Feed materials are selected that are gaseous at the temperatures $(20-300 \mathrm{C})$ of the flow control system used to inject the material into the TIS and to fully dissociate at the operating temperatures of the TIS (1000-2000 C). Since the feed material is introduced at a controlled rate from an external injection system rather than through diffusion from solid target material, as in on-line process, effusive delay times in the TIS can be delineated from of the diffusion release times associated with the target material.

Interest in generating radioactive ion beams (RIBs) of ${ }^{17} \mathrm{~F}$ and ${ }^{18} \mathrm{~F}$ for astrophysics research [1] at the Holifield Radioactive Ion Beam Facility (HRIBF) [2] and the use of the electron beam plasma target/ion source (EBPTIS) for their generation was the motivating factor for studying the effusive flow characteristics of fluorine and fluoride compounds formed in this type of ion source. The TIS used in these studies is very similar to sources described previously [3]. When employed in on-line RIB generation, nuclear reaction products, created by high energy light ion bombardment of thick target materials are first diffused from the target material located inside a target material reservoir and then transported to the ionization chamber of a TIS through a vapor transport tube where they undergo electron impact ionization. For the HRIBF TIS used in these experiments, the target material reservoir and transport tube are resistively heated to temperatures in excess of $1000 \mathrm{C}$. Ion beams extracted from the ionization chamber are accelerated to energies of $20-60 \mathrm{keV}$. All measurements were made first with the target material reservoir empty and then with fiberous $\mathrm{Al}_{2} \mathrm{O}_{3}$ target material with a nominal fiber diameter of $3 \mu \mathrm{m}$ [4]. Ionization efficiencies for Xe during operation were typically $15 \%$, falling to $6.5 \%$ with $\mathrm{Al}_{2} \mathrm{O}_{3}$ present due to the increased vapor pressure of the $\mathrm{SiO}_{2}$ contaminants in the target material.

\section{FLOW MEASUREMENT AND CONTROL APPARATUS}

During RIB generation, the flow rates of the radioactive species are typically $10^{8}$ to $10^{13}$ atoms $/ \mathrm{s}$, which, for most cases, is insufficient to form a monolayer on the interior surfaces of the TIS. Therefore, studies must be made in a flow-rate regime comparable to those that occur in typical on-line operations for RIB generation. Toward this end, we have developed a simple, static-vacuum, flow measurement system, capable of directly measuring gaseous flows down to $\sim 10^{12}$ particles/s and well defined, reproducible feed material flow-rates down to $\sim 10^{10}$ particles/s. Molecular gases were introduced into the TIS from a feed material reservoir, maintained at pressures

"The submitted manuscript has been authored by a contractor of the U.S. Government under contract No. DEAC05-96OR22464. Accordingly, the U.S. Government retains a nonexclusive royalty-free license to publish or reproduce the published form of this contribution, or allow others to do so, for U.S. Government purposes." 


\section{DISCLAIMER}

Portions of this document may be illegible in electronic image products. Images are produced from the best available original document. 


\section{DISCLAIMER}

This report was prepared as an account of work sponsored by an agency of the United States Government. Neither the United States Government nor any agency thereof, nor any of their employees, makes any warranty, express or implied, or assumes any legal liability or responsibility for the accuracy, completeness, or usefulness of any information, apparatus, product, or process disclosed, or represents that its use would not infringe privately owned rights. Reference herein to any specific commercial product, process, or service by trade name, trademark, manufacturer, or otherwise does not necessarily constitute or imply its endorsement, recommendation, or favoring by the United States Government or any agency thereof. The views and opinions of authors expressed herein do not necessarily state or reflect those of the United States Government or any agency thereof. 


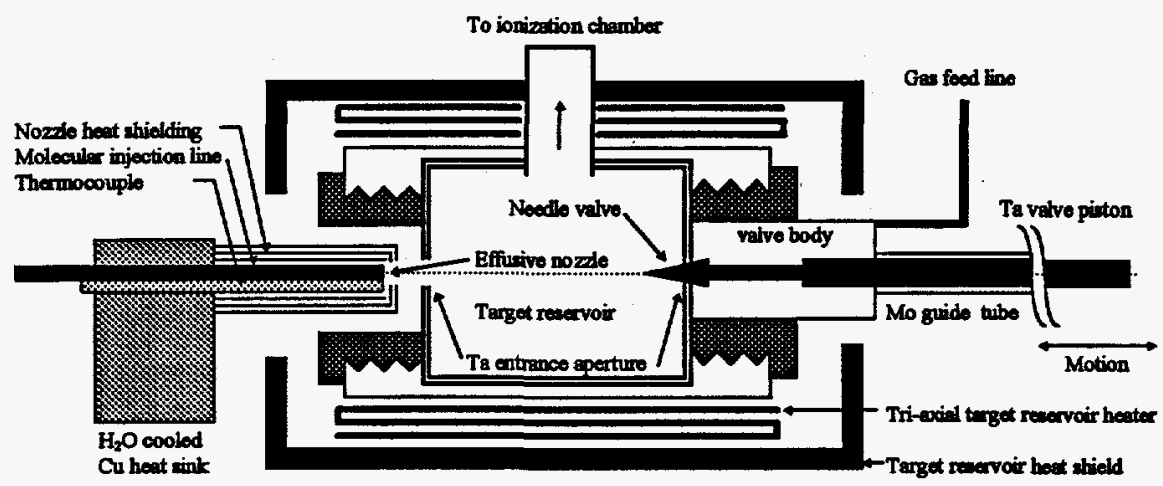

FIGURE 1. A schematic illustration of the high temperature valve assembly used in measuring th effusive flow delay times (Right) and the sample injection system used for the introduction of intact molecules into the TISS for the determination of ionization efficiencies (Left).

between 1 and 1000 Torr. The reservoir provided a static pressure for controlling the feed-rates of the material in question through a set of calibrated and variable leaks; the feed material is then directed into a small flow measurement volume, maintained at $1-100 \mathrm{mT}$ Trr, or fed directly into the TIS by means of a two-way valve. The measurement volume is independently evacuated and can be isolated to time the rate-of-rise in pressure due to gas flow through the leak valves. If $j_{m v} N_{m v}(t), n_{m v}(t), P_{m v}(t)$, $T_{m v}$ are, respectively; the feed material leak rate, number of molecules, number density, pressure and temperature in the flow measurement volume, then

$$
j_{m v}=\frac{d N_{m v}}{d t}=V_{m v} \frac{d n_{m v}}{d t}=\frac{V_{m v}}{k T_{m v}} \cdot \frac{d P_{m v}}{d t} .
$$

Capacitance manometers are used to measure pressures in the feed material reservoir and the flow measurement volume. The background out-gassing rate of the measurement volume was typically less than $10^{12}$ molecules/s.

The magnitudes of feed material flow-rates from the flow-rate measurement system were confirmed with a calibrated Xe leak supplied by VTI Scientific [5] and with a He leak detector, calibrated against two standard leaks. Measured flow-rates always varied less than $10 \%$ through a given leak and agreed, typically, with calibrated leak rates to within $\sim 1 \%$. The system was also tested by flowing $\mathrm{Ne}, \mathrm{Ar}, \mathrm{Kr}, \mathrm{SF}_{6}, \mathrm{CF}_{4}$ and $\mathrm{CCl}_{2} \mathrm{~F}_{2}$ through the calibrated leak and each species was seen to obey the theoretically predicted $\mathrm{M}^{-1 / 2}$ flow dependence to within a few percent. In addition, a linear relationship was observed between the feed material reservoir pressure and ion current for each of the species in the flow regime studied. After measuring the flow rate of the desired species in the $10^{12}$ to $10^{13}$ molecules/s range, the reservoir pressure was reduced to provide scaled flowrates of the species of interest as low as $10^{10}$ molecules/s.

\section{EFFUSIVE DELAY TIME MEASUREMENTS}

The mean effusive delay time for a given species within the TIS can be determined by either rapidly establishing or interrupting the flow into the TIS while monitoring the time dependence of the mass selected ion current. A high temperature valve, close coupled to the target material reservoir, is employed to minimize contributions of particle delay from the injection feed line to the measured delay functions of the TIS. The valve must have a minimal influence on the target material reservoir temperature when either opened or closed to avoid sources or sinks of sample particles. The valve assembly is schematically depicted in Fig. 1. The valve seat occurs between a carbonized tapered Ta needle and a thin Ta plate. The needle and supporting shaft are thermally isolated from the external environment and maintained at the target reservoir temperature by radiant heat. The valve assembly is enclosed in the heat shielding of the TIS where W-Re thermocouples continuously monitor the temperatures of the target reservoir, valve body, valve needle and Mo guide tube. By displacing the piston, the conductance between the valve body and target reservoir can be varied over several orders of magnitude.

The delay function is defined as the time response of the mass selected ion current, $I(t)$, to changes in the flux of the particles entering the TIS, $j(t)$. If $j(t)$ is small enough so that ionization conditions are not perturbed, 
the total number of sample particles in the TIS, $N(t)$, is proportional to $I(t)$ [6]. Furthermore, if $j(t)$ can be rapidly changed with respect to the delay times of the species within the TIS, the mean particle delay time can be determined from the measurement. By applying formalism from the kinetic theory of gases to the dynamic particle balance within the TIS, under molecular flow conditions, we obtain,

$$
I(t) \propto N(t)=e^{-\alpha s} \int_{-\infty}^{t} j\left(t^{\prime}\right) e^{\alpha t^{\prime}} d t^{\prime} \text { where } \alpha=1 / \tau
$$

Here $\tau$ is the mean delay time for the sample particle in the TIS. Eq. 2 is readily solved for the case where $j(t)$ is rapidly reduced from $j_{1}$ to $j_{0}$ at time $t_{1}$

$$
N(t)=\frac{j_{1}-j_{0}}{\alpha} e^{-\alpha\left(t-t_{1}\right)}+\frac{j_{0}}{\alpha}+k
$$

where $k-0$ if the valve closes rapidly with respect to $\tau$. Similarly if $j(t)$ is rapidly raised from $j_{0}$ to $j_{l}$ at time $t_{1}$ the number of particles is given by [7]

$$
N(t)=\frac{j_{1}}{\alpha}-\frac{j_{1}-j_{0}}{\alpha} e^{-\alpha(t-\xi)}-k .
$$

Effusive delay time measurements were made by recording $I(t)$ for the sample species and the background Ta current during each valve opening and closing. Initial tests with $\mathrm{Xe}$ show excellent agreement between the measured delay function and the delay function predicted from evacuation of the TIS volume, assuming conductance limited flow through the extraction opening ( $\tau$-volume/conductance). Fluorine delay functions were studied by introducing $\mathrm{SF}_{6}$ to create a source of atomic $\mathrm{F}$ within the valve body which was maintained at temperature above of $1200 \mathrm{C}$ to allow complete thermal dissociation of this molecule [8]. In addition to $F^{+}$, the mass spectrum of the extracted beams also contained $\mathrm{AlF}^{+}$and $\mathrm{BeF}_{2}{ }^{+}$with no $\mathrm{SF}_{\mathrm{x}}^{+}$present. The metal fluorides, likely result from the interaction of atomic fluorine with impurities which were always present in the TIS over $\sim 1000$ hours of operation in spite of several TIS cleanings and replacement of the feed system.

An example of the measured delay function characteristic of AlF is shown in Fig. 2; The solid curves are fits to the data derived by use of Eqs. 3 and 4. The delay times were studied as a function of $\mathrm{SF}_{6}$ flow into the TIS; a very clear flow independent regime was found for equivalent $\mathrm{F}$ flow-rates of less than $\sim 2 \mu \mathrm{A}$. These results are shown in Fig. 3. The addition of the $\mathrm{Al}_{2} \mathrm{O}_{3}$ target material to the target reservoir had little effect on the observed delay times. In addition to $\mathrm{SF}_{6}, \mathrm{CCl}_{2} \mathrm{~F}_{2}$ and

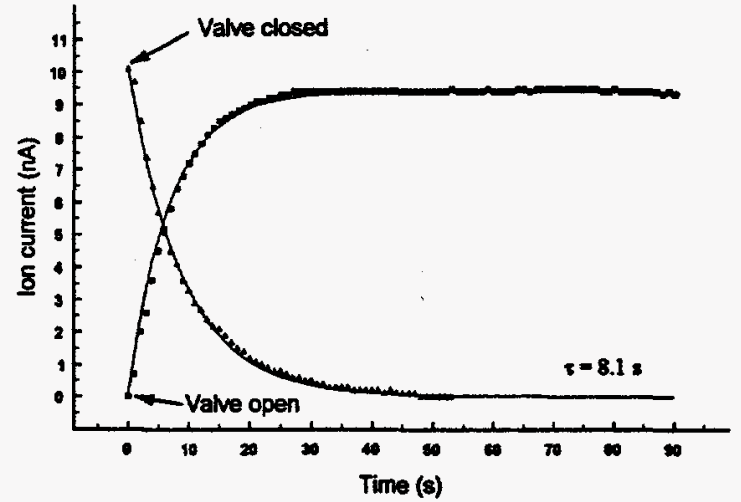

FIGURE 2. Example delay time measurement for AF' from the TIS with Eq. 3 and 4 fitted to the data to determine the effusive delay time.

$\mathrm{CF}_{4}$ were also employed as means for supplying atomic F. The delay times for $\mathrm{F}^{+}, \mathrm{AlF}^{+}$and $\mathrm{BeF}_{2}{ }^{+}$from $\mathrm{CCl}_{2} \mathrm{~F}_{2}$ and $\mathrm{SF}_{6}$ were nearly identical while the delays for $\mathrm{F}^{+}$and $\mathrm{CF}_{\mathrm{x}}^{+}$from $\mathrm{CF}_{4}$ were considerably shorter. This can be explained on the basis of complete molecular dissociation of both $\mathrm{SF}_{6}$ and $\mathrm{CCl}_{2} \mathrm{~F}_{2}$ in the TIS while $\mathrm{CF}_{4}$ remains intact at the TIS operating temperatures [8].

\section{IONIZATION EFFICIENCY MEASUREMENTS}

Experience shows that physically attaching the gas feed line to the high temperature TIS results in significant conductive heat transfer to the feed line and consequently causes thermal dissociation of $\mathrm{SF}_{6}$ in relatively cool regions of the line (650-1200 C). This causes substantial and indeterminal losses of throughput due to chemical reaction of the reactive $F$ feed material with the walls of the feed tube. To circumvent this problem, we designed, developed and characterized an effusive-flow nozzle, separated from the high temperature target material reservoir by a gap of $4.2 \mathrm{~mm}$, which operates at temperatures below the threshold for molecular dissociation of $\mathrm{SF}_{6}(650 \mathrm{C})$. The nozzle permits injection of known fractions of intact molecular feed gases into the TIS. The $\mathrm{SF}_{6}$ molecules will fully dissociate at the temperatures of the TIS (1200-1400 C) [8]. Ionization efficiencies of $F$ are determined by comparing the mass analyzed ion currents with the total flux of $F$ delivered to the TIS by the $\mathrm{SF}_{6}$ gas, after correcting for losses to the vacuum system due to the injection gap. A schematic representation of the water cooled injection system is shown in Fig. 1.

Fractional losses of particles across the injection gap into the surrounding vacuum system were determined for two limiting cases: (i) particles which pass through the injection gap in the vacuum system are lost (maximum loss scenario) and (ii) molecules have a maximum 


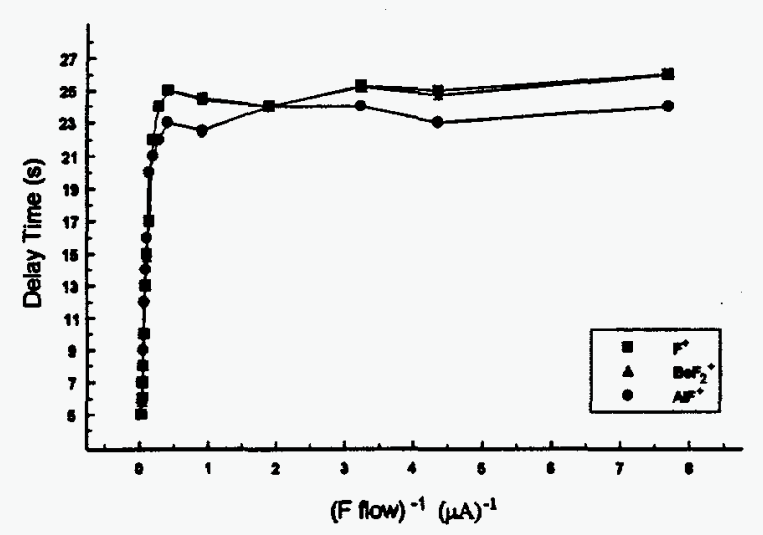

FIGURE 3. Fluorine/fluoride delay times plotted versus the reciprocal of the equivalent $\mathrm{F}$ flow rate introduced into the TIS using $\mathrm{SF}_{6}$ as the carrier species at a target reservoir temperature of $1525 \mathrm{C}$.

probability of reentering the TIS through the injection aperture since no adsorption losses are assumed (minimum loss scenario). The maximum fractional loss, case (i), can be determined either by use of Monte Carlo effusive flow simulations of the feed-line/TIS injection system [6] or by employing analytical approximations derived from the kinetic theory of gases under Knudsen flow conditions [9]. The minimum fractional loss, case (ii), can be determined from experiment by introducing an equivalent mass and flow-rate of noble gas (no dissociation/adsorption losses) into the TIS and monitoring the ion current yielded with the injection gap open and then with the gap sealed. Once the two limiting extremes of the fractional loss are determined, the ionization efficiency can be calculated for each case from the measured ion currents. If the dimensions and placement of the nozzle are chosen correctly, the uncertainty in the ionization efficiency measurement can be made reasonably small. For these experiments, the minimum possible transmission of particles into the TIS from the feed system (maximal loss) was calculated and found to vary from 3 to $13 \%$, depending on the size of the entrance aperture used. The maximum possible transmission (minimal loss) was measured using noble gases and also found to vary from 10 to $40 \%$ depending on the size of the entrance aperture.

Ionization efficiencies were determined from the mass selected ion currents of $F$ and the fluorides extracted from the EPBTIS over equivalent $F$ flow-rates from 1 to $10 \mu \mathrm{A}$, correcting for particle transmission across the injection gap. The ionization efficiencies were found to be: F: 1.6 to $0.8 \%$; AlF: 1.8 to $0.9 \%$; and $\mathrm{BeF}_{2}: 1.4$ to $0.7 \%$, respectively. The addition of $\mathrm{Al}_{2} \mathrm{O}_{3}$ target material had the effect of reducing both the Xe and all fluoride efficiencies by a factor of $\sim 2.3$ ostensibly due to a persistent vapor load on the TIS.

\section{DISCUSSION}

The measured delay functions for both fluorides and Xe can be represented theoretically using Eqs. 3 and 4. The measured mean delay times for evacuation of Xe from the TIS agrees with the theoretical values. Examination of the measured fluoride delay times versus flow-rate clearly shows an independent flow-rate regime below $2 \mu \mathrm{A}$ of $\mathrm{F}$ flow and a linear dependence on (flow rate) ${ }^{-1}$ at feed rates above $2 \mu \mathrm{A}$. This behavior is predicted from the adsorption kinetics of a surface which has reached saturation at $2 \mu \mathrm{A}$ of flow [10]. The delay. times for $\mathrm{F}$ and $\mathrm{BeF}_{2}$ are identical thus, $\mathrm{BeF}_{2}$ appears to be the dominant transport agent for the $F$ through the TIS. The measured $\mathrm{AlF}$ and $\mathrm{BeF}_{2}$ delay times of $25 \mathrm{~s}$ are in approximate agreement with the delay time expected from adsorption enthalpy data $(3.2,3.1 \mathrm{eV}$, respectively), mean number of wall collisions within the TIS $(\chi-1000)$ and the estimated effective temperature of the TIS [11].

The ionization efficiency measurement technique detailed in this report is also able to measure efficiencies for reactive $F$ at flow rates which have been shown, from delay time studies, to preclude surface saturation effects and are therefore in a flow-rate regime comparable to those for RIB generation. Because many molecules decompose at temperatures available in RIB TISs, the techniques and apparatus used to measure delay times and ionization efficiencies of fluorine are applicable to other RIB TIS systems and for other RIB species. These methods provide direct measurements of delay times and ionization efficiencies for candidate RIB species independent of the release characteristics of the target allowing delineation of effusive and diffusive flow processes when compared with on-line studies.

\section{REFERENCES}

1. M.S. Smith, NIMB 99 (1995) 349

2. D.K. Otsen, Nuci. Instr. Meth A328 (1993) 303

3. G.D. Alton, Rev. Sci. Instrum. 65 (1994) 2012

4. Alumina fiber supplied by RATH Preformance fibers, Wilmington, MA. USA

5. Vacuum Technology Incorperated, Oak Ridge, TN 37832 USA

6. R.F. Welton, G.D. Alton, A Piotrowski, S.N. Murray, Rev. Sci. Instrum. 67 (1996) 1670

7. R. Kirchner, Nucl. Instrum. Methods B70 (1992) 186

8. Calculations were made by using The Thermocale which is a product of the Royal Institute of Technology Stockholm, Sweden and HSC Software from Outokumpu Research Oy, Pori, Finland

9. A Roth, 'Vacuum Technology', Elsevier, New York (1990)

10. V. Ponec, Z. Knor and S. Cerny, 'Adsorption on solids', Butterworth \& Co, London (1974)

11. G. Bolbach, J.C. Blais, and A. Marilier, Sur. Sci, 90 (1979) 65 